\title{
Biphasic Polycondensation of 4-Bromo-2,6-dimethylphenol Using Silica Gel as a Promoter
}

\author{
By Yuji SHIBASAKI, ${ }^{1, *}$ Maiko TAKAHASHI, ${ }^{1}$ Manabu TANAKA, ${ }^{2}$ \\ Junko N KONDO, ${ }^{2}$ and Yoshiyuki OISHI ${ }^{1}$
}

Poly(2,6-dimethyl-1,4-phenylene ether) (PPE) was successfully prepared by the biphasic polymerization of 4-bromo-2,6dimethylphenol (BDMP) with the use of silica gel as a promoter at $20^{\circ} \mathrm{C}$ under air. Silica gel was hydrolyzed with alkaline to afford water-soluble siloxane oligomers, which acted as an effective phase transfer agent for the biphasic polymerization of BDMP. The pure PPE was easily separated from the siloxane oligomers by the ordinal reprecipitation process, and the oligomers were reassembled into silica gel by the condensation of silanol groups in an acidic medium. The morphologies of the reassembled silica gel are worm-like and spherical with wormholes in it.

KEY WORDS: Poly(2,6-dimethyl-1,4-phenylene ether) / 2,6-Dimethylphenol / Phase Transfer Catalyst / Biphasic Polymerization /

Silica Gel / Siloxane / Alkaline Hydrolysis /

Organic-aqueous interfacial synthesis is an attractive alternative to conventional homogeneous synthesis. ${ }^{1}$ By using a phase transfer catalyst (PTC) to transfer the substrates from an inorganic phase into an organic one, interfacial synthesis permits convenient recovery of the catalyst and reaction product. At the same time, the ability of the PTC to take up the substrates and confine them in the interfacial region makes it possible to promote the desired reaction faster and more selectively than in homogeneous processes. Thus, many PTCpromoted polymerizations have been developed, where much milder conditions and much more rapid polymerization were realized. ${ }^{2}$ Generally speaking, biphasic polymerization proceeds by the use of cationic or nonionic PTCs such as ammonium and phosphonium salts or crown ethers, which are commercially available oil-derived materials. Silica gel, industrially prepared from silicon dioxide, is most commonly encountered in our everyday life as a desiccant, and in the laboratory as the stationary phase in chromatography. Siliconebased PTC has been developed and commercialized, in which hydrophilic side chains such as oxyethylene units, derived from oil, are introduced onto a polysiloxane backbone. ${ }^{3}$ It is known that silica gel can be alkaline hydrolyzed, resulting in alkali metal silicates, whose solution contains a variety of anionic silicate species in dynamic equilibrium. ${ }^{4}$ In the presence of an organic base such as tetrapropylammonium salt, the silicate anion forms several specific silicate cages, i.e., a curved hydrophobic $\mathrm{SiO}_{2}$ surface encompasses the organic molecule with all hydroxyl groups of the silicate pointing outward. ${ }^{5}$ Thus, a bifunctional interface with an outer hydrophilic and an inner hydrophobic surface is created.

Poly(2,6-dimethyl-1,4-phenylene ether) (PPE) is a wellknown engineering plastic that can be catalytically prepared from 2,6-dimethylphenol with oxygen via an oxidative coupling polycondensation. ${ }^{6}$ This polymer is usually used in a modified form, e.g., a blend of PPE with polyamide, polyolefin, or polystyrene, in order to decrease its processing temperature. ${ }^{7}$ PPE is also available by the PTC polycondensation of 2,6dimethylphenol $^{8}$ or 4-bromo-2,6-dimethylphenol (BDMP) ${ }^{9}$ with and without copper catalyst, but all of these are the result of utilizing an oil-derived PTC.

We expect that if silica gel was added into an alkali metal BDMP solution in water/organic medium, the organic molecule would form inside the silica cages, followed by consecutive coupling to give PPE. We here report the first non-oilderived PTC polymerization, the polycondensation of BDMP with silica gel as a PTC.

\section{EXPERIMENTAL}

\section{Reagents}

Silica gel (Wako gel C300: grain size 45-75 $\mu \mathrm{m}, 100-200$ mesh) was purchased from Wako Pure Chemicals and used as received. All other reagents and solvents were used as received.

\section{Polymerization}

Method A. Into a sample vial containing a magnetic stir bar were placed sodium hydroxide $(1.6 \mathrm{~g}, 40 \mathrm{mmol})$, BDMP $(0.38 \mathrm{~g}, 1.9 \mathrm{mmol})$, and distilled water $(5 \mathrm{~mL})$. The solution was vigorously stirred under air until the monomer was completely dissolved. Silica gel $(0.0038 \mathrm{~g}, 1 \mathrm{wt} \%$ to the monomer) was added, and the mixture was stirred at $20^{\circ} \mathrm{C}$ for $0.5 \mathrm{~h}$ under air. Toluene $(5 \mathrm{~mL})$ was then added, and the polymerization was kept for $12 \mathrm{~h}$ at this temperature. The resulting viscous emulsion was poured into methanol $(50 \mathrm{~mL})$ containing concentrated hydrogen chloride $(1 \mathrm{~mL})$ to precipitate the polymer. The precipitate was collected and washed with methanol, water, $1 \mathrm{~N}$ hydrogen chloride solution in water, and methanol. It was then dried, dissolved in chloroform, and 
reprecipitated in methanol $(50 \mathrm{~mL})$ containing concentrated hydrogen chloride $(1 \mathrm{~mL})$ through filter paper to give a white fibrous polymer $(0.17 \mathrm{~g}, 76 \%$ yield).

Method B. Into a sample vial containing a magnetic stir bar were placed sodium hydroxide $(1.6 \mathrm{~g}, 40 \mathrm{mmol})$, BDMP $(0.38 \mathrm{~g}, 1.9 \mathrm{mmol})$, and distilled water $(5 \mathrm{~mL})$. The solution was vigorously stirred under air until the monomer was completely dissolved. Toluene $(5 \mathrm{~mL})$ and silica gel $(0.0038 \mathrm{~g}$, $1 \mathrm{wt} \%$ to the monomer) were added consecutively, and the mixture was stirred at $20^{\circ} \mathrm{C}$ for $12 \mathrm{~h}$ under air. The other protocols were the same as in Method A. A white fibrous polymer was obtained $(0.18 \mathrm{~g}, 79 \%$ yield $)$.

\section{Reassembly of Silica Gel}

Typical procedures were as follows. After the polymerization using silica gel $(0.38 \mathrm{~g}, 100 \mathrm{wt} \%)$ in method $\mathrm{B}$, concentrated hydrogen chloride was added into the polymerization solution until the $\mathrm{pH}$ of the solution become almost 1 . The heterogeneous mixture was stirred for $12 \mathrm{~h}$, and then poured into methanol. The precipitate was collected, washed with methanol, water, $1 \mathrm{~N}$ hydrogen chloride solution in water, methanol, and dried. The resulting solid was dissolved in chloroform, and filtered to remove PPE. The solid on the filter was carefully washed with chloroform, and dried in vacuo to give a white solid $(0.32 \mathrm{~g}, 83 \%$ yield $)$.

\section{Oligomerization of BDM for NMR Measurements}

To characterize the polymer chain ends, a relatively low molecular weight polymer was prepared in $1 \mathrm{~h}$ by method B. The corresponding polymer was obtained as a white powder ( $73 \%$ yield, $M_{\mathrm{n}}=5,300, M_{\mathrm{w}} / M_{\mathrm{n}}=1.8$ ).

\section{Polymerization of BDMP by Reassembled Silica Gel}

The reassembled silica gel was used instead of the commercially available silica gel (Wako gel C300) for the polymerization of BDMP by method B. A white fibrous polymer was obtained ( $86 \%$ yield, $M_{\mathrm{n}}=72,700, M_{\mathrm{w}} / M_{\mathrm{n}}=$ 2.4).

\section{Measurements}

${ }^{1} \mathrm{H}$ NMR spectra were recorded on a Bruker AC400 NMR spectrometer. Deuterated chloroform was used as the solvent for ${ }^{1} \mathrm{H}$ NMR measurements with tetramethylsilane as an internal standard. Number-average and weight-average molecular weights $\left(M_{\mathrm{n}}\right.$ and $\left.M_{\mathrm{w}}\right)$ were estimated by gel permeation chromatography (GPC) with a Tosoh HLC 8120 GPC system with polystyrene gel columns (TSKgel) calibrated by polystyrene standard samples at $40^{\circ} \mathrm{C}$ in chloroform. Thermogravimetric analysis (TGA) and differential scanning calorimetry (DSC) were performed with a Seiko TG/DTA320 and a Shimadzu DSC190 at a heating rate of $10^{\circ} \mathrm{C} / \mathrm{min}$ under nitrogen, respectively. Scanning electron micrographs were collected on a Hitachi S-2300 at $15 \mathrm{kV}$ accelerating voltage. $\mathrm{N}_{2}$ adsorption-desorption isotherms were measured by SA-3100 systems, and pore-size distributions were determined by the Barrett-Joyner-Halenda (BJH) method.

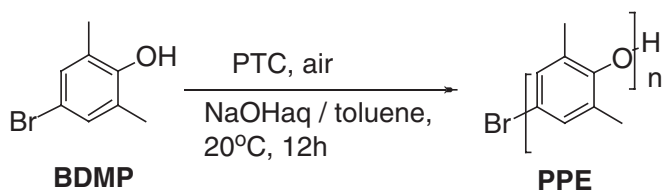

Scheme 1.

Table I. Biphasic polymerization of BDMPa

\begin{tabular}{ccccccc}
\hline Run & $\begin{array}{c}\text { Silica gel } \\
/ \text { wt }^{\text {b }}{ }^{\mathrm{a}}\end{array}$ & $\begin{array}{c}\text { TBAHS } \\
/ \text { wt } \%^{\mathrm{b}}\end{array}$ & $\begin{array}{c}\mathrm{NaOH} \\
\text { /equiv }^{\mathrm{c}}\end{array}$ & $\begin{array}{c}\text { Yield } \\
/ \%\end{array}$ & $M_{\mathrm{n}}{ }^{\mathrm{d}}$ & $M_{\mathrm{w}} / M_{\mathrm{n}}{ }^{\mathrm{d}}$ \\
\hline 1 & 0 & 0 & 20 & 12 & 2,700 & 1.5 \\
2 & 0 & 20 & 20 & 24 & 20,200 & 2.3 \\
3 & 20 & 0 & 20 & 74 & 92,800 & 4.4 \\
4 & 1 & 0 & 20 & 79 & 79,700 & 3.7 \\
5 & 0.5 & 0 & 20 & 90 & 85,100 & 7.0 \\
6 & 1 & 0 & 15 & 59 & 22,700 & 2.8 \\
\hline
\end{tabular}

apolymerization conditions: BDMP $1.9 \mathrm{mmol}, \mathrm{H}_{2} \mathrm{O} 5 \mathrm{~mL}$, toluene $5 \mathrm{~mL}$, at $20^{\circ} \mathrm{C}$ for $12 \mathrm{~h}$ under air. Method $\mathrm{B}$ was applied. ${ }^{b}$ Weight $\%$ to BDMP. ${ }^{c}$ Equivalent to $\mathrm{OH}$ group of BDMP. ${ }^{\mathrm{d}}$ Determined by $\mathrm{GPC}\left(\mathrm{CHCl}_{3}\right.$, PSt standards).

\section{RESULTS AND DISCUSSION}

As described in the Introduction, silica gel is easily decomposed in alkaline solution to give water-soluble siloxane oligomers, which can be reassembled into silica gel by adjusting the $\mathrm{pH}$ of the solution. To confirm whether silica gel functions as a PTC, the biphasic polymerization of BDMP was performed with and without silica gel under air (Table I). When the polymerization was conducted in toluene/alkaline biphasic solution without silica gel, only oligomeric products were obtained in poor yield (Run 1). A typical oil-derived PTC, tetrabutylammonium hydrogen sulfate (TBAHS), gave a polymer in $24 \%$ yield (Run 2). In contrast, the addition of the same amounts of silica gel gave a polymer with a higher molecular weight in good yield (Run 3 ). These results clearly suggested that silica gel, a non-oil derived material, acts as a powerful PTC under these conditions. In addition, only $0.5 \mathrm{wt} \%$ silica gel was found to effectively promote the polymerization of BDMP (Run 5). The concentration of the alkaline solution affected the rate of the biphasic polymerization of BDMP, which is generally observed in this type of reaction. ${ }^{8 b}$

Silica gel is transformed into various types of water-soluble siloxane oligomers, whose structures are dynamically interconverted with each other especially in the presence of organic molecules. ${ }^{5}$ Thus, determining the conditions for the alkaline hydrolysis of silica gel is important for the subsequent biphasic polymerization of BDMP. We examined two methods of hydrolysis for the polymerization. In Method A, silica gel is hydrolyzed in an alkaline medium by itself. In Method B, silica gel is added into a toluene/alkaline biphasic solution, so that the silica gel is alkaline hydrolyzed at the interface. In this case, silica gel is transformed into water-soluble siloxane oligomers, which can include toluene as a guest molecule. 
Table II. Biphasic polymerization of BDMP by using silica gel as PTC

\begin{tabular}{rrrrrr}
\hline Run & Method $^{\mathrm{b}}$ & $\begin{array}{c}\text { Silica gel } \\
/ \text { wt } \%^{\mathrm{c}}\end{array}$ & $\begin{array}{c}\text { Yield } \\
/ \%\end{array}$ & $M_{\mathrm{n}}{ }^{\mathrm{d}}$ & $M_{\mathrm{w}} / M_{\mathrm{n}}{ }^{\mathrm{d}}$ \\
\hline 1 & $\mathrm{~A}$ & 1 & 76 & 50,700 & 3.1 \\
2 & $\mathrm{~A}$ & 20 & 76 & 70,100 & 4.3 \\
3 & $\mathrm{~A}$ & 50 & 37 & 76,200 & 8.8 \\
4 & $\mathrm{~A}$ & 80 & 29 & 15,300 & 1.9 \\
5 & $\mathrm{~A}$ & 100 & 43 & 32,700 & 3.3 \\
6 & $\mathrm{~B}$ & 1 & 79 & 79,700 & 3.7 \\
7 & $\mathrm{~B}$ & 20 & 74 & 98,800 & 4.4 \\
8 & $\mathrm{~B}$ & 50 & 84 & 112,600 & 5.4 \\
9 & $\mathrm{~B}$ & 80 & 84 & 84,400 & 7.7 \\
10 & $\mathrm{~B}$ & 100 & 86 & 63,600 & 4.9 \\
\hline
\end{tabular}

a Polymerization conditions: BDMP $1.9 \mathrm{mmol}, \mathrm{H}_{2} \mathrm{O} 5 \mathrm{~mL}$, toluene $5 \mathrm{~mL}$, at $20^{\circ} \mathrm{C}$ for $12 \mathrm{~h}$ under air. 'b Method A: Silica gel was added into the BDMP solution in $\mathrm{NaOH}$ (aq.) After stirring the solution for $15 \mathrm{~min}$, toluene was added. Method B: Silica gel was added into the biphasic solution of BDMP in $\mathrm{NaOH}$ (aq.) and toluene. ${ }^{c}$ Weight \% to BDMP. ${ }^{d}$ Determined by GPC $\left(\mathrm{CHCl}_{3}\right.$, PSt standards)

Table II summarizes the results of the polymerization. In Method A, high molecular weight polymers were obtained in good yield when the polymerization was conducted with less than $20 \mathrm{wt} \%$ of silica gel as a PTC (Runs 1 and 2). However, when using larger amounts of silica gel, both the yields and molecular weights of the polymer decreased (Runs 3, 4, and 5). In contrast, these were clearly improved in Method B (Runs 6 to 10). These results indicated that the water-soluble siloxane oligomers obtained in Method B were favorable for the biphasic polymerization of BDMP. The siloxane oligomers containing toluene as guest molecules might favorably encapsulate BDMP and its oligomers.

In Method B, there is no significant difference in the molecular weights of the resulting polymer. This value, however, gradually increased to 112,600 , and decreased after that. This might be due to the effect of the size and number of micelles in the solution. Therefore, in the polymerization with $50 \mathrm{wt} \%$ silica gel, the size and number of the micelles created from the resulting siloxane oligomers should be the best for the fast propagation of PPE.

To understand the polymerization behavior in more detail, the number-average molecular weights $\left(M_{\mathrm{n}}\right)$ of the polymer were plotted against the polymerization time as shown in Figure 1. The polymerization was performed by Method B. The polymerization mixture became an emulsion after more than about $6 \mathrm{~h}$. A waxy mixture resulted after $12 \mathrm{~h}$ which made it difficult to isolate the product polymer. Therefore, the polymerization time was set to be $12 \mathrm{~h}$ in this experiment.

The $M_{\mathrm{n}}$ linearly increased with polymerization time. Theoretically, the degree of the polymerization $X_{\mathrm{n}}$ is expressed as

$$
X_{\mathrm{n}}=\mathrm{C}_{0} k t+1
$$

where $\mathrm{C}_{0}, k$, and $t$ are initial concentration of the monomer, rate constant of the polymerization, and polymerization time, respectively. Therefore, the rate constant $k$ was estimated to be $0.0425 \mathrm{~s}^{-1}$ from the slope in Figure 1 .

Figure 2 depicts a) the ${ }^{1} \mathrm{H}$ NMR spectrum of the PPE

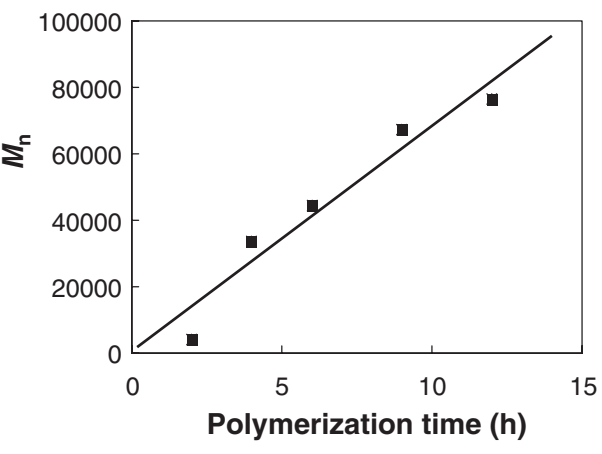

Figure 1. $M_{\mathrm{n}}$ vs. polymerization time by the use of $1 \mathrm{wt} \%$ of silica gel as a PTC (Run 6, Table II)

prepared in Method B, and b) the expanded aromatic region. Two typical signals are observed at 2.08 and $6.47 \mathrm{ppm}$, which are assigned to the methyl and aromatic protons ( $a$ and $b$ ), respectively. In the expanded spectrum, terminal aromatic protons $d$ of the PPE are observed at $6.36 \mathrm{ppm}$. Hydroxyl protons in the polymer-chain-end are also observed at $4.24 \mathrm{ppm}$, which was broadened by the addition of deuterium oxide into this solution, indicating the active hydrogens. The integration ratio of $d$ and $c$ is 2.00:0.86, which is close to the theoretical values of $2: 1$. From the integration ratio of the signals of the polymer-chain-end to the polymer backbone, the molecular weight of the polymer was calculated to be $M_{\mathrm{n}(\mathrm{NMR})}=5,640$; thus $X_{\mathrm{n}}=47$. This is in good agreement with the molecular weight estimated by GPC measurements $\left(M_{\mathrm{n}}=5,300\right)$. The TGA and DSC analysis of the prepared PPE were performed under nitrogen, in which $5 \mathrm{wt} \%$ loss temperature $\left(T_{\mathrm{d} 5}\right)$ and glass transition temperature $\left(T_{\mathrm{g}}\right)$ were 435 and $220^{\circ} \mathrm{C}$, respectively, which were in good agreement with the values of the commercially available PPE $\left(T_{\mathrm{d} 5}=430^{\circ} \mathrm{C}\right.$, $T_{\mathrm{g}}=215^{\circ} \mathrm{C}$ ). These results also support the satisfactory formation of pure PPE.

Water-soluble siloxane oligomers were reassembled into silica gel after the polymerization by adjusting the $\mathrm{pH}$ of the solution. Figure 3 depicts the TGA profiles of a) the originally used silica gel (Wako gel C300) and b) reassembled silica gel under nitrogen. In the original silica gel, $7 \%$ weight-loss was observed after heating to $800^{\circ} \mathrm{C}$, which could be attributed to absorbed water. In the reassembled silica gel under the same heating conditions, $16 \%$ weight-loss was observed. This could be both absorbed water and the water produced by condensation between silanol functions. No organic residues were found in the elemental analysis of the reassembled silica gel. ${ }^{10}$ It is noticeable that no significant weight-loss was observed at more than $150^{\circ} \mathrm{C}$, suggesting that the reassembled silica gel has no organic parts.

To investigate the reassembled silica gel in more detail, nitrogen absorption-desorption experiments were performed. Figures 4 and 5 represent the profiles and pore diameter distributions. Table III summarizes the BET surface areas and pore diameters of the various silica gels obtained from Figures 4 and 5. Originally used silica gel has a BET surface area of $353 \mathrm{~m}^{2} / \mathrm{g}$ and pore diameter of $7.63 \mathrm{~nm}$. In contrast, the 
a)

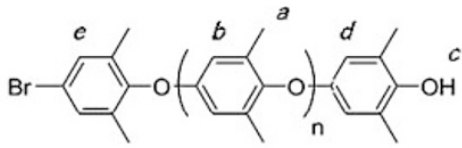
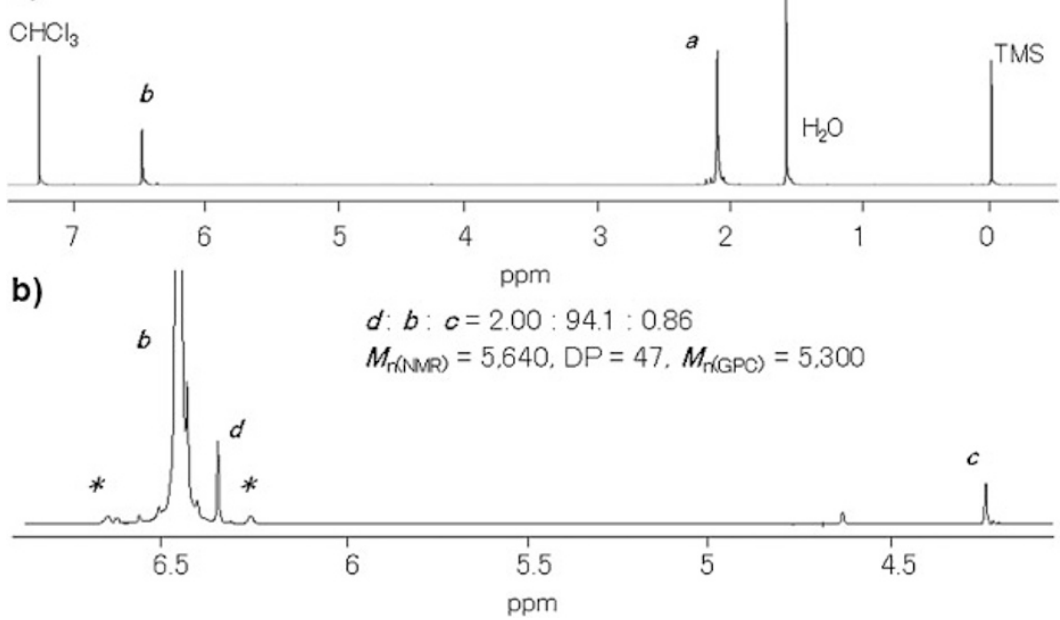

Figure 2. a) ${ }^{1} \mathrm{H}$ NMR spectrum $\left(\mathrm{CDCl}_{3}, 20^{\circ} \mathrm{C}\right)$ of PPE prepared by the biphasic polymerization of BDMP and b) the expanded spectrum. Signals marked with $*$ are spinning side bands.

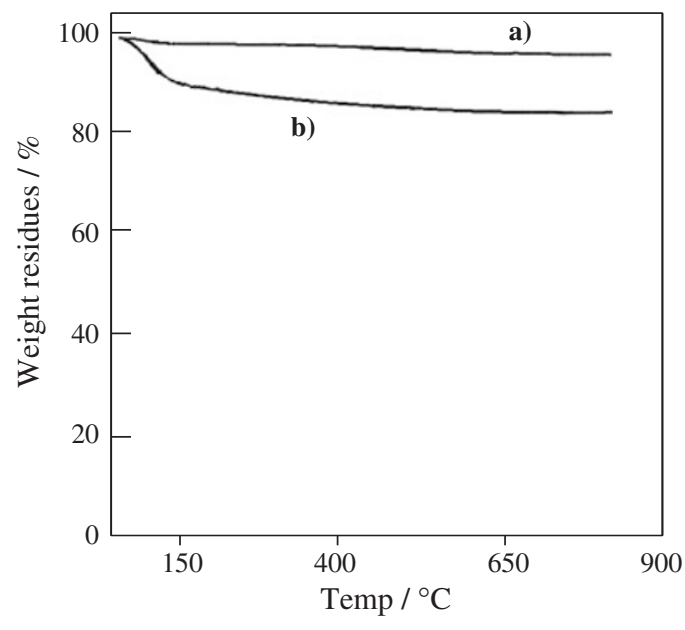

Figure 3. TGA profiles of a) original and b) reassembled silica gels under nitrogen.

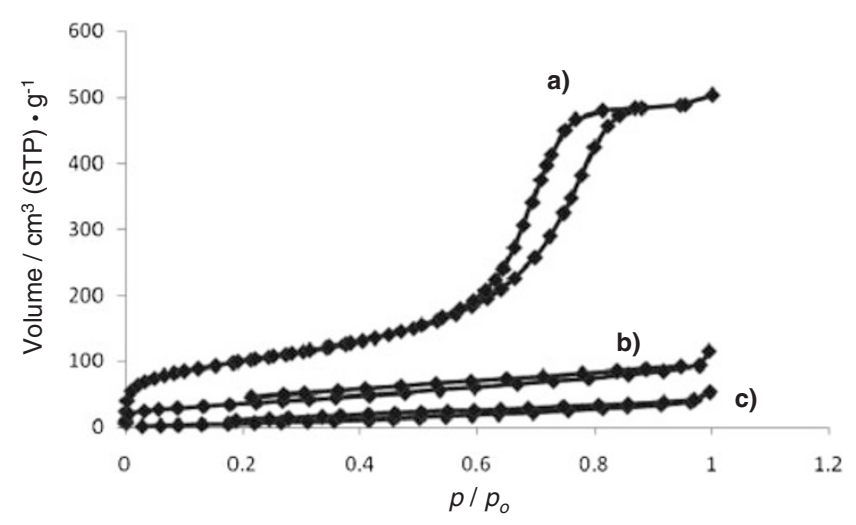

Figure 4. $\mathrm{N}_{2}$ gas adsorption-desorption isotherms of (a) original silica gel (Wako gel C300), (b) reassembled silica gel (run 8 in Table II), and (c) reassembled silica gel (run 10 in Table II).

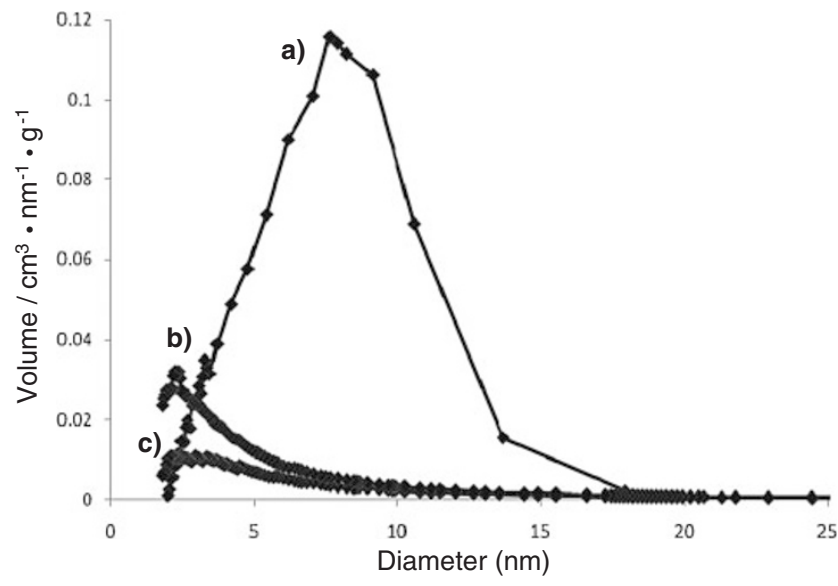

Figure 5. The pore size distribution of (a) original silica gel (Wako gel C300), (b) reassembled silica gel (run 8 in Table II), and (c) reassembled silica gel (run 10 in Table II). Pore size distribution was calculated using BJH analysis.

Table III. BET surface areas and pore diameters of original and reassembled silica gels

\begin{tabular}{cccc}
\hline Run & Silica gel & $\begin{array}{c}\mathrm{BET} \text { surface } \\
/ \mathrm{m}^{2} \cdot \mathrm{g}^{-1}\end{array}$ & $\begin{array}{c}\text { Pore diameter } \\
/ \mathrm{nm}\end{array}$ \\
\hline 1 & Original $^{\mathrm{a}}$ & 353 & 7.63 \\
2 & Reassembled $_{50} \mathrm{~b}$ & 39.0 & 2.43 \\
3 & Reassembled $_{100}{ }^{\mathrm{C}}$ & 126 & 2.23 \\
\hline
\end{tabular}

a Originally used silica gel (Wako gel C300). ${ }^{\text {b}}$ Reassembled silica gel used for the polymerization of BDMP (Run 8 , Table II). ${ }^{\mathrm{C}}$ Reassembled silica gel used for the polymerization of BDMP (Run 10, Table II).

reassembled silica gels have less BET surface areas of 39 to $126 \mathrm{~m}^{2} / \mathrm{g}$ and smaller pore diameters of 2.2 to $2.4 \mathrm{~nm}$. These results suggested that the silica gel was first decomposed into siloxane oligomers, which functioned as a PTC for the polymerization of BDMP. 
a)

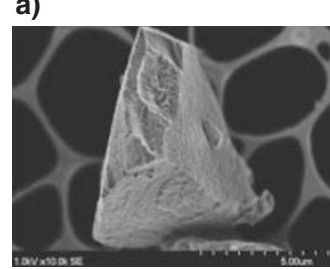

c)

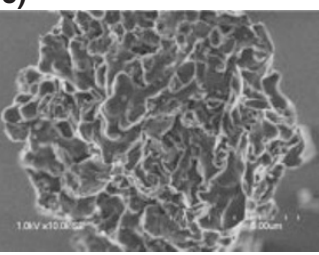

b)

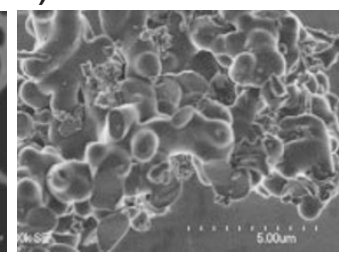

d)

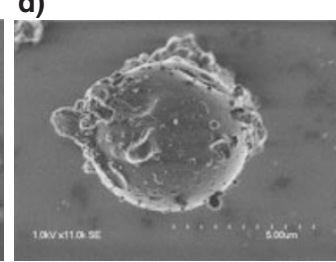

e)

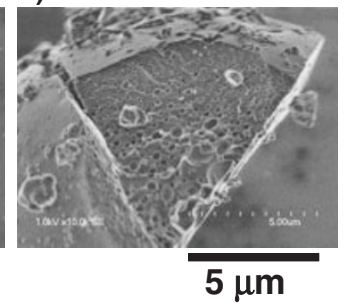

Figure 6. Scanning micrographic images of a) original silica gel, b) reassembled silica gel obtained in the polymerization of run 8 in Table II, and c)-e) reassembled silica gel obtained in the polymerization of run 10 in Table II.

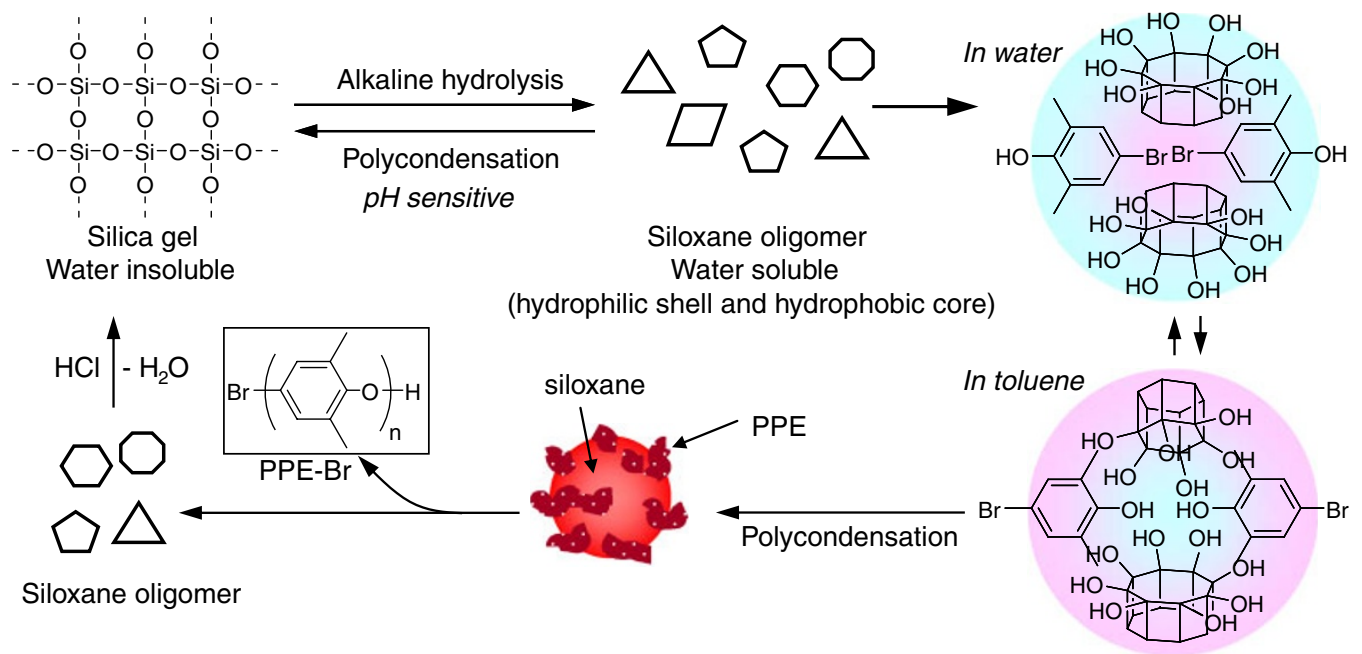

Figure 7. Plausible mechanism for the biphasic polymerization of BDMP with silica gel.

Figure 6 depicts the scanning micrographic (SEM) images of a) original silica gel, b) reassembled silica gel obtained in the polymerization of run 8 in Table II $(50 \mathrm{wt} \%$ silica gel was used), and c)-e) reassembled silica gel obtained in the polymerization of run 10 in Table II $(100 \mathrm{wt} \%$ silica gel was used). The typical grain size of the original silica gel was about $5 \mu \mathrm{m}$ as shown in Figure 6a, and the reassembled silica gels have smaller grain sizes. The reassembled silica gel obtained after the polymerization of BDMP (50 wt \% silica gel) took similar worm-like forms (Figure 6b), but there were two types of morphologies in the reassembled silica gel $(100 \mathrm{wt} \%$ silica gel); one is worm-like forms as seen in Figure $6 \mathrm{c}$, and the other one is sphere-like structures (Figure 6d and 6e). Spherical silica gel has a smooth surface (Figure 6d), but inside of the sphere there are many wormhole structures (Figure 6e). The size of the pore is around $150 \mathrm{~nm}$, and the distribution is large. Because of this, no significant capillary concentration phenomena are observed in Figure $4 \mathrm{~b}$ and $4 \mathrm{c}$. Thus we cannot conclude if there are penetrated pores inside the sphere.
In order to know the durability of the catalyst, the silica gel reassembled after the polymerization of run 10 in Table II was reused for the polymerization under the same conditions as run 6 in Table II. As a result, a polymer with $M_{\mathrm{n}}$ of 72,700 $\left(M_{\mathrm{w}} / M_{\mathrm{n}}=2.4\right)$ was obtained in $86 \%$ yield, indicating that the reassembled silica has a similar ability to catalyze the polymerization of BDMP.

Figure 7 depicts a plausible mechanism of this polymerization. First, the silica gel is decomposed with alkaline solution to give water-soluble siloxane oligomers. Because these siloxane oligomers have hydrophobic cores, the monomer is absorbed in the structure, and the polymerization takes place in such concentrated conditions. Thus, the biphasic polymerization of BDMP efficiently proceeds under mild conditions, giving high molecular weight PPE with corresponding structures. The siloxane oligomers can be reacted with each other by changing the $\mathrm{pH}$ to give reassembled silica gel, which was easily separated from the PPE by filtration. 


\section{SUMMARY}

We have demonstrated the first biphasic polymerization of BDMP promoted by silica gel, a non-oil derived material. High molecular weight PPE was easily obtained at ambient temperature under air. The kinetics of the polymerization obeyed theoretical equations generally observed in condensation polymerizations. The resultant PPE was carefully characterized to be the pure PPE free from silicon atoms. The reassembled silica gel has no contamination from PPE, and the morphology was unique, e.g., worm-like and spheres containing wormholes, which were observed in SEM measurements. The reassembled silica gel also functioned as a PTC for the polymerization of BDMP with similar activity of the commercially available silica gel.

Acknowledgment. This work was partially supported by Tokuyama Science Foundation, and by an Industrial Technology Research Grant 2003 from NEDO (New Energy and Industrial Technology Development Organization) of Japan (ID 03A23010c).

Received: August 27, 2008 Accepted: September 30, 2008 Published: November 12, 2008

\section{REFERENCES}

1. W. P. Weber and G. W. Gokel, "Phase Transfer Catalysis in Organic Synthesis" Springer Verlag, 1977.
2. a) P. M. Morgan, "Condensation Polymers by Interfacial and Solution Methods" Inerscience, New York, 1965.

b) V. V. Korshak and V. A. Vasnev, in "Comprehensive Polymer Science" G. Allen, Jr. and C. Bevington, Ed., Pergamon Press, 1989, vol. 5, p 167.

3. K. Sakuda, Mater. Technol., 17, 323 (1999).

4. G. Engelhardt and D. Michael, in "High-Resolution Solid-State NMR of Zeolites and Related Systems" John Wiley and Sons, Condon, 1987, chapter III, p75.

5. a) S. D. Kinrade, C. T. G. Knight, D. L. Pole, and R. T. Syvitski, Inorg. Chem. 37, 4272 (1998).

b) C. E. A. Kirschhock, R. Ravishnkar, F. Verspeurt, P. J. Grobet, P. A. Jacobs, and J. A. Martens, J. Phys. Chem. B, 103, 4965 (1999).

6. a) D. M. White, in "Comprehensive Polymer Science" G. Allen, Jr. and C. Bevington, Ed., Pergamon Press, 1989, vol. 5, p 473. b) A. S. Hay, J. Polym. Sci., Part A: Polym. Chem., 36, 505 (1998).

7. O. Olabisi, L. M. Robeson, and M. T. Shaw, "Polymer-polymer miscibility" Academic Press, New York, 1979.

8. a) Y. Zhong, C. F. Adams, and P. K. Lim, Ind. Eng. Chem. Res., 34, 1529 (1995).

b) Y. M. Chung, W. S. Ahn, and P. K. Lim, Appl. Catal., A, 192, 165 (2000).

c) P. Gamez, J. A. P. P. van Dijk, W. L. Driessen, G. Challa, and J. Reedijk, Adv. Synth. Catal., 344, 890 (2002).

d) K. Saito, T. Tago, T. Masuyama, and H. Nishide, Angew. Chem., Int. Ed., 43, 730 (2004).

9. a) G. D. Staffin and C. C. Price, J. Am. Chem. Soc., 82, 3632 (1960). b) C. C. Price and N. S. Chu, J. Polym. Sci., 61, 135 (1962).

c) V. Percec and T. D. Shaffer, J. Polym. Sci., Polym. Lett. Ed., 24, 439 (1986).

d) K. Mühlbach and V. Percec, J. Polym. Sci., Polym. Chem. Ed., 25, 2605 (1987).

e) V. Percec and J. H. Wang, J. Polym. Sci., Polym. Chem. Ed., 29, 63 (1991).

10. a) A. S. Araujo and M. Jaroniec, Thermochim. Acta, 363, 175 (2000). b) Nakajima, M. Matsuoka, S. Mishima, and I. Matsuzaki, Nippon Kagaku kaishi, 11, 972 (1994). 